\title{
Objective tinnitus due to essential palatal myoclonus
}

\author{
Daryus Heydari ${ }^{1}$, Levent Eker $^{2}$ and Hakan Kayali ${ }^{3 *}$ \\ ${ }^{1}$ Departments of Neurology, Private Afiyet Hospital, Istanbul, Turkey \\ ${ }^{2}$ Departments of Otorhinolaryngology, Private Afiyet Hospital, Istanbul, Turkey \\ ${ }^{3}$ Departments of Neurosurgery, Private Afiyet Hospital, Istanbul, Turkey
}

\begin{abstract}
Objective tinnitus can be heard by both the patient him or herself and examiner. Objective tinnitus can be treated in many cases by medicines like muscle relaxants, anti-convulsatants, barbiturates, benzodiazepines, and anti-cholinerjics or can be treated by surgically. To the best of our knowledge in medical treatment there is not use of gabapentin for medical treatment of objective tinnitus. In this report a woman $40 \mathrm{y}$-old has for a long time objective tinnitus was presented.. She was treated by gabapentin and full recovery was observed.
\end{abstract}

\section{Introduction}

Objective tinnitus is much less then the subjective tinnitus and can be heard by both the patient and examiner. There are three forms of objective tinnitus.

1. Myoclonic tinnitus, 2. Vascular tinnitus, 3. Tinnitus depends on patulous Eustachian tube.

Careful history taking and physical examination, audiologic, medical and radiologic evaluations are needed for the suitable diagnosis and treatment for each type of objective tinnitus [1].

Incidence of objective tinnitus has reported over 17\% among 211 tinnitus patients who evaluated by Yoo $\mathrm{HJ}$ et al. [2].

\section{Myoclonic tinnitus}

It can be subdivided to palatal myoclonic tinnitus and middle ear myoclonic tinnitus [1].

\section{Palatal tyoclonic tinnitus}

Palatal myoclonic tinnitus is rare and it can be appear by the voluntary or involuntary contraction of palatal muscle. There are two types including essential (EPT) and symptomatic (SPT) types.

The etiology of either form is still remains unclear. Essential form may be arised by dysfunction and symptomatic may be arised by a lesion of the connections between dentate nucleus, red nucleus and inferior olivary nuclei, which is called the Guillain-Mollaret triangle [1].

Although symptomatic form which develops secondary tumourous, ischemic or degenerative lesions of the brainstem or the cerebellum, essential form has not structural lesion [3]. The theories about the origin of the clicking sound heard by the patient and the outside examiner vary. Some authors believe in that this sound comes from contraction of the stapedial or tensor tympani muscles and the others favour the opening and closing of the eusachian tube or contraction of the muscles tensor veli palatini and levator veli palatini which are attached to it [4].
In EPT, the tensor veli palatini muscle (TVP), which is innervated by cranial nerve $\mathrm{V}$, is generally thought to be the culprit. It is hypothesized that the ear clicks are produced by contractions of the TVP muscle that opens the eustachian tube, causing a sudden decrease of the surface tension within the tube and all imaging and laboratory investigations are normal.

In SPT, ear clicks are rarely present, and palatal movements, which rarely bring a patient to medical attention, are part of a constellation of clinical findings, including dysarthria, nystagmus and ataxia. The levator veli palatini muscle (LVP), which is innervated by cranial nerves IX and X, is responsible for the rhythmic movements of the soft palate. Imaging changes in SPT include olivary pseudohypertrophy and possibly evidence of the causative lesion in the dentato-olivary tract [5].

Although the diagnosis of objective tinnitus as such is easy, but the myoclonus is not so easily seen. The rhytm is mostly at a rate of 1-2 cliks/second but it may vary, the palatal myoclonus is not seen in all cases by the naked eye. Briefly, It is emphasized that the objective tinnitus formed in the palatal myoclonus is the result of contraction of M.stapedius, M.tensor tympani, M.tensor veli palatini and M. levator veli muscles [4].

\section{Middle ear myoclonic tinnitus}

It is very rare. It has been suggested that the crackling or buzzing sounds are caused by repetetive and abnormal tensor tympani and stapedial muscle contractions [6]. These myoclonic movements are caused by segmental myoclonus involving brainstem innervated muscles and infectious and demyelinating diseases, vascular diseases, anxiety, trauma or neoplastic disorders can be roled on etiology of segmental myoclonus [6-8].

${ }^{*}$ Correspondence to: Hakan Kayali, Department of Neurosurgery, Private Afiyet Hospital, Namık Kemal Mah. Sutcu Imam Cad. No: 8 Umraniye-Istanbul,Turkey, E-mail: hakankayali@hotmail.com

Key words: objective tinnitus, treatment, gabapentin

Received: July 04, 2020; Accepted: September 21, 2020; Published: September 28,2020 


\section{Vascular tinnitus}

There are mainly three etiologies. 1. Arterial Causes, 2. Venous causes and 3. Vascular neoplasm of the skull base and temporal bone. Arterio-venous malformations, intracranial arteriovenous fistulae and aneurysm, atherosclerotic carotid artery disease increased cardiac output due to anemia, thyrotoxicosis or pregnancy, high jugular bulb, benign intracranial hypertension, venous hum are mainly arterial or venous vascular reasons [1].

\section{Patulous eusthachian tube}

There are several etiologies. Loss of tissue within the cartiloginous portion of eusthachian tube by weight loss, high-dose oral contraseptives and estrogen therapy. The other reasons are atrophy or scarring of eusthachian tube or nasopharynx caused by adenoidectomy, radiotherapy or iatrogenic traumas leads to dysfunction of eusthachian tube $[9,10]$.

\section{Case presentation}

A fourty year-old female patient admitted to us with the tinnitus can be heard by herself for a year. Cerebral computed tomography, Cranial Magnetic Resonance Imaging, Cranial MR Angiography and otorhinnolaryngologic examinations include tympanic membran pressure had been recognized as normal in previous examinations. She had been treated by benzodiazepines, barbiturates, myorelaxants. But when these treatments did not benefit, finally she had been advised to psychiatry so she had begun to use antidepressants for six months but no curable. So she had left to use antidepressants by herself for last two months.

In our examination, she was considered an Essential form of Palatal Myoclonic tinnitus and treated by Gabapentin $600 \mathrm{mg}$ b.i.d. At the end of one month treatment period full recovery was observed both of the patient and us.

\section{Discussion}

Objective tinnitus can be cured in many cases but, if it is not been diagnosed correctly it can be distressing. In medical treatment anticonvulsatants, myorelaxants, benzodiazepines, barbiturates, and anticholinergic agents can be used. The palatal myoclonus is not seen in all cases by the naked eye [4]. We also did not see palatal myoclonus by naked eye. On the other hand all clinical and laboratory findings were normal expect objective tinnitus so she was considered an Essential form of Palatal Myoclonic tinnitus.

To the best of our knowledge gabapentine was not used in medical treatment of objective tinnitus up to date. As the other medicines were used before were insufficient, in this case gabapentin $600 \mathrm{mg}$ b.i.d was used for one month and full recovery was observed.

Although the exact mechanism of action of Gabapentin is still unknown most probably its' anticonvulsatant effect play role on the musculus TVP which innervated by the cranial nerve V, like Carbamezepine effect on the treatment of trigeminal neuralgia.

Of course gabapentin treatment needs to using on more and more patients to see the exact results on objective tinnitus, however it may be considerable an alternative treatment instead of the other anticonvulsants or on drug resistance objective tinnitus.

\section{References}

1. Shi Nae Parki Hangyang (2016) Objective Tinnitus. Med Rev 36: 99-108.

2. Yoo HJ, Park SN, Kim DK, Park KH, Kim MJ, et al. (2011) Incidence and clinical characteristics of patients with tinnitus according to diagnostic classification. Korean $J$ Otorhinolaryngol-Head Neck Surg 54: 392-398.

3. Duschel G, Mischeke G, Schenk E, Shulte-Monting J, Luking CH (1990) Symptomatic and essential rhythmic palatal myoclonus. Brain 113:1645-1672.

4. Rahko T, Hakkien VX (1979) Carbamazepine in the treatment of objective myoclonus tinnitus. J Laryngol Otol 93: 123-127.

5. Zadikoff C, Lang AE, Klein C (2006) The 'essentials' of essential palatal tremor. A reappraisal of the nosology. Brain 129: 832-940.

6. Lee GH, Bae SC, Jin SK, Park KH, Yeo SW, et al. (2012) Middle ear myoclonus associated with forced eyelid closure in children diagnosis and treatment outcome. Laryngoscope 122: 2071-2075.

7. Marchiando A, Per-Lee JH, Jackson RT (1983) Tinnitus due to idiopathic stapedial muscle spasm. Ear Nose Throat J 62: 8-13.

8. Park SN, Bae SC, Lee GH, Song JN, Park KH, et al. (2013) Clinical characteristics and therapeutic response of objective tinnitus due to middle ear myoclonus: A large case series. Laryngoscope 123: 2516-2520.

9. Plate S, Johnsen NJ, Nodskov Pedersen S, Thomsen KA (1979) The frequency of patulous eustachian tubes in pregnancy. Clin Otolaryngol Allied Sci 4: 393-400.

10. Pulec JL (1967) Abnormally patent eustachian tubes. Treatment with injection of polytetrafluoroethylene (teflon) paste. Laryngoscope 77:1543-1554.

Copyright: (C2020 Heydari D. This is an open-access article distributed under the terms of the Creative Commons Attribution License, which permits unrestricted use, distribution, and reproduction in any medium, provided the original author and source are credited. 